\title{
Functional neuroimaging: a physiological perspective
}

\author{
Ai-Ling Lin ${ }^{1,2 *}$, Jia-Hong Gao ${ }^{3}$, Timonthy Q. Duong ${ }^{1,4,5,6,7}$ and Peter T. Fox ${ }^{1,2,4,6,7}$ \\ 1 Research Imaging Institute, University of Texas Health Science Center, San Antonio, TX, USA \\ 2 Department of Psychiatry, University of Texas Health Science Center, San Antonio, TX, USA \\ ${ }^{3}$ Department of Psychiatry and Behavioral Neuroscience, and Brain Research Imaging Center, University of Chicago, Chicago, IL, USA \\ ${ }^{4}$ Department of Physiology, University of Texas Health Science Center, San Antonio, TX, USA \\ ${ }^{5}$ Department of Ophthalmology, University of Texas Health Science Center, San Antonio, TX, USA \\ ${ }^{6}$ South Texas Veterans Health Care System, Department of Veterans Affairs, San Antonio, TX, USA \\ 7 Department of Radiology, University of Texas Health Science Center, San Antonio, TX, USA
}

\section{Edited by:}

Anna Devor,

University of California San Diego, USA

\section{Reviewed by:}

Richard Buxton,

University of California San Diego, USA

Joseph Mandeville, Massachusetts

General Hospital, USA

David Boas, Massachusetts General Hospital, USA; Massachusetts Institute of Technology, USA; Harvard Medical School, USA

\section{*Correspondence:}

Ai-Ling Lin, Research Imaging Center, School of Medicine, The University of Texas Health Science Center, 7703

Floyd Curl Drive, San Antonio, TX

78229, USA.

e-mail: lina3@uthscsa.edu
Metabolic physiology and functional neuroimaging have played important and complementary roles over the past two decades. In particular, investigations of the mechanisms underlying functional neuroimaging signals have produced fundamental new insights into hemodynamic and metabolic regulation. However, controversies were also raised as regards the metabolic pathways (oxidative vs. non-oxidative) for meeting the energy demand and driving the increases in cerebral blood flow (CBF) during brain activation. In a recent study, with the concurrent functional MRIMRS measurements, we found that task-evoked energy demand was predominately met through oxidative metabolism (approximately 98\%), despite a small increase in cerebral metabolic rate of oxygen (12-17\%). In addition, the task-induced increases in CBF were most likely mediated by anaerobic glycolysis rather than oxygen demand. These observations and others from functional neuroimaging support the activation-induced neuron-astrocyte interactions portrayed by the astrocyte-neuron lactate shuttle model. The concurrent developments of neuroimaging methods and metabolic physiology will also pave the way for the future investigation of cerebral hemodynamics and metabolism in disease states.

Keywords: functional neuroimaging, $\mathrm{CBF}, \mathrm{CMRO}_{2}, \mathrm{ANLS}$ model, neurodegenerative disorders
The quest to understand the functional organization of the brain has occupied mankind for more than a century. The close relationship between cerebral blood flow $(\mathrm{CBF})$ and brain function was first observed in the late nineteenth century by the Italian physiologist, Mosso (1881). Mosso recorded pulsations from the forearm and the brain, showing stronger brain pulsations during cognitive events, such as hearing the bells from his church and performing mental arithmetic (Posner and Raichle, 1994). These studies suggested that measurement of CBF might be an important way to assess brain function during mental activity. Roy and Sherrington (1890), two distinguished British physiologists, further characterized the close relationship between brain function and CBF. They attributed task-induced vasodilation to an increased demand for cerebral metabolism in response to neuronal activity. The Roy-Sherrington principle has been interpreted to mean that $\mathrm{CBF}$ changes reflect a tight coupling between cellular energy requirements and vascular delivery of glucose and oxygen. Roy and Sherrington's observation of focally increased blood flow during brain functional activation has been replicated thousands of times by a myriad of techniques, beginning early in the last century (Fulton, 1928).

Whole-brain measurements of CBF, oxygen metabolism (cerebral metabolic rate of oxygen, $\mathrm{CMRO}_{2}$ ) and glucose metabolism $\left(\mathrm{CMR}_{\mathrm{Glc}}\right)$ also began appearing in the early part of the twentieth century (Schmidt and Kety, 1947; Landau et al., 1955; Sokoloff et al., 1977). Collectively, these studies demonstrated that: the brain's resting-state energy demand is high (although the human brain is only $2 \%$ of body's weight, it consumes $20 \%$ of the body's oxygen and 25\% body's glucose; Sokoloff et al., 1977); resting-state brain energy production is provided almost exclusively by glucose oxidation (approximately 90\%; Siesjo, 1978); and, basal CBF and $\mathrm{CMRO}_{2}$ are tightly coupled across brain regions (A linear CBF$\mathrm{CMRO}_{2}$ relationship was observed with a slope of approximately 0.97; Fox and Raichle, 1986).

Studies using emerging, pre-imaging radiotracer techniques convincingly demonstrated that brain blood flow is dramatically increased by increased partial pressure of carbon dioxide $\left(\mathrm{PaCO}_{2}\right)$ and by decreased partial pressure of oxygen $\left(\mathrm{PaO}_{2}\right)$, a form of cerebrovascular autoregulation (Kety and Schmidt, 1948). These observations provided strong support for the Roy and Sherrington hypothesis, as $\mathrm{CO}_{2}$ is the primary "chemical product" of glucose oxidation, and extended the hypothesis to include substrate $\left(\left[\mathrm{O}_{2}\right]\right)$ availability as a potent vascular regulator. With the advent of autoradiographic methods (Schmidt and Kety, 1947; Landau et al., 1955; Sokoloff et al., 1977) and non-invasive radiotracer imaging methods (Reivich et al., 1979; Raichle et al., 1983), blood flow and glucose consumption could be measured regionally, allowing the Roy and Sherrington hypothesis to be further explored. As expected, task performance reliably elicited large, highly focal increases in CBF (Fox et al., 1988) and $\mathrm{CMR}_{\mathrm{Glc}}$ (Phelps and Mazziotta, 1985; Fox et al., 1988). The observed increases in CBF and $\mathrm{CMR}_{\mathrm{Glc}}$ were similar in magnitude, typically in the range of $30-50 \%$, apparently supporting the Roy and Sherrington hypothesis that neural activity focally increased metabolic rate and thereby increased blood flow. 
The first imaging-based measurements of $\mathrm{CMRO}_{2}$ during task performance were reported in the early 1980s, using $15-\mathrm{O}$ positron emission tomography (PET) (Frackowiak et al., 1980; Mintun et al., 1984). In two different brain systems (visual and somatosensory), Fox et al., observed that task-induced increases in $\mathrm{CMRO}_{2}$ were much lower than those in $\mathrm{CBF}$ or $\mathrm{CMR}_{\mathrm{Glc}}$ (Fox and Raichle, 1986; Fox et al., 1988). The $\mathrm{CMRO}_{2}$ shortfall during focal neuronal activation, in fact, caused a local oxygen surplus, with the oxygen extraction fraction (OEF) falling from a resting value of approximately $40 \%$ to a task-state value of approximately $20 \%$. These findings clearly contradicted the Roy and Sherrington hypothesis. Since glucose can be metabolized by either oxidative or non-oxidative (i.e, lactate-producing) pathways and since the increase of $\mathrm{CMRO}_{2}$ was minimal, Fox and colleagues suggested that: (i) the glucose is predominately metabolized by anaerobic glycolysis; (ii) the energy demand associated with neuronal activation is small (as opposed to resting-state demand) and glycolysis alone may provide the energy needed for the transient changes in brain activity; and (iii) $\mathrm{CBF}$ response must be regulated by factors other than oxidative metabolism and total energy demand. The observation that the stimulus-evoked increase in glucose consumption observed with PET is at least partially non-oxidative has been confirmed with ${ }^{1} \mathrm{H}$ NMR spectroscopic (MRS) measurements of tissue lactate concentration [Lac] (Prichard et al., 1991; Frahm et al., 1996). The increase in lactate production (i.e., non-oxidative metabolism) was found in later studies to be a modulator of CBF increase (Mintun et al., 2004). Such uncoupling of CBF and $\mathrm{CMRO}_{2}$ is the basis for the blood oxygenation level-dependent (BOLD) functional MRI (fMRI) contrast (Kwong et al., 1992; Ogawa et al., 1992). These PET and MRS observations raise a general question: does the evoked neuronal activity drive oxidative or non-oxidative metabolism?

To further clarify this issue, we performed concurrent fMRI and ${ }^{1} \mathrm{H}$ MRS measurements to identify the relationship between task-evoked increases in $\mathrm{CBF}, \mathrm{CMRO}_{2},[\mathrm{Lac}]$ and ATP production $\left(\mathrm{J}_{\text {ATP }}\right)$ during graded visual stimulation $(4,8$, and $16 \mathrm{~Hz}$; $4 \mathrm{~min}$ for each condition) (Lin et al., 2010). Percent changes (\% $\%$ ) in $\mathrm{CBF}, \mathrm{CMRO}_{2}$ and $[\mathrm{Lac}]$ varied with frequency, with $\% \Delta \mathrm{CBF}$ and $\% \Delta[\mathrm{Lac}]$ peaking at $8 \mathrm{~Hz}$, while $\% \Delta \mathrm{CMRO}_{2}$ reached a maximum at $4 \mathrm{~Hz}$ (Vafaee and Gjedde, 2000; Lin et al., 2008). The magnitude of $\% \Delta \mathrm{CBF}(57.1-65.1 \%)$ and $\% \Delta[\mathrm{Lac}](31.3-50.0 \%)$ were much larger than that of $\% \Delta \mathrm{CMRO}_{2}(12.2-17.0 \%)$. As a result, $\% \triangle \mathrm{CBF}$ was tightly coupled with lactate production rate $\left(\mathrm{J}_{\mathrm{Lac}}\right.$; [Lac] divided by stimulation period; $r=0.91, P<0.001$ ) (Gjedde, 1997; Lin et al., 2010), but negatively correlated with $\% \Delta \mathrm{CMRO}_{2}$ $(r=-0.64, P=0.024) . \mathrm{J}_{\mathrm{ATP}}$, determined with changes in $\mathrm{CMRO}_{2}$ and $\mathrm{J}_{\mathrm{Lac}}$ by a stoichiometric relationship, was found predominately contributed by oxidative metabolism (approximately $98 \%$ ) at each stimulus condition. Consequently, $\% \Delta \mathrm{J}_{\text {ATP }}$ was linearly correlated to $\% \Delta \mathrm{CMRO}_{2}(r=1.00, P<0.001)$. Similar findings were also reported by PET studies (Gjedde, 1997). The fMRI-MRS observations confirm that (i) CBF response to neuronal activity is driven more by anaerobic glycolysis, rather than oxygen demand, and (ii) energy demand is predominately met through oxidative metabolic pathway regardless the $\mathrm{CMRO}_{2}$ increases are much lower than those of [Lac].
The collective evidence from the functional imaging literature (PET, fMRI, and MRS) has forced the development of alternatives to the Roy-Sherrington hypothesis. Of these, the astrocyte-neuron lactate shuttle (ANLS) model is the most conceptually evolved and widely accepted (Pellerin and Magistretti, 1994). The ANLS model posits a cooperation between neurons and astrocytes in meeting the activation-induced needs both for energy production and for neurotransmitter production. Upon neuronal firing, glucose is taken up by both neurons and astrocytes. The majority of the glucose is taken up by the astrocytes and the remainder by neurons. Glucose metabolism in neurons is small but entirely aerobic, to support neurotransmission (Brand, 2005; Hyder et al., 2006). Astrocytic glucose consumption, on the other hand, is large but much less energetically efficient by virtue of being predominately anaerobic. Astrocytic glycolysis (2 ATP) is used to support $\mathrm{Na}^{+} / \mathrm{K}^{+}$ion pumping and glutamate(Glu)-glutamine( $\mathrm{Gln}$ ) conversion. Lactate generated by astrocytic glycolysis is eventually transported to neurons as fuel, but with some loss into the circulation, which increases hyperemia (Mintun et al., 2004; Hyder et al., 2006).

The fMRI-MRS observations, described above (Lin et al., 2010), are in line with the ANLS model. First, CBF increases are not regulated by oxidative metabolism. $\% \Delta \mathrm{CBF}$ and $\% \Delta \mathrm{CMRO}_{2}$ are negatively correlated. In contrast, non-oxidative metabolism is a more likely candidate. $\% \Delta \mathrm{CBF}$ and $\% \Delta \mathrm{J}_{\mathrm{Lac}}$ are positively correlated. But other factors (e.g., $\mathrm{Ca}^{2+}, \mathrm{K}^{+}$and adenosine signaling pathway) may also play a role. Second, the increases in $\mathrm{CMR}_{\mathrm{Glc}}$ are for purposes other than energy demand, e.g., for astrocyte-mediated neurotransmitter recycling, with the evidence being that increases in $[\mathrm{Lac}]$ are far more than that of $\mathrm{CMRO}_{2}$. Third, the two metabolic pathways (oxidative and non-oxidative) are co-existent, dissociable, and serve different purposes in maintaining neuronal functions during visual stimulation. Oxidative metabolism is predominately neuronal and supports ATP production for the release of neurotransmitters; with the evidence being that the energy demand $\left(\mathrm{J}_{\text {ATP }}\right)$ was predominately (approximately 98\%) met through oxidative metabolism in all stimulus conditions. Non-oxidative metabolism, on the other hand, mainly occurs in astrocytes to support the Glu-Gln conversion and lactogenesis-mediated hyperemia. Since astrocytic lactate is eventually taken up into the tricarboxylic acid cycle as a fuel substrate by neurons, it has to be pointed out that oxidative metabolism is expected to increase as neuronal activation continues. In support of this formulation, prolonged visual stimulation $(>20 \mathrm{~min})$ has been reported to induce gradually rising levels of $\mathrm{CMRO}_{2}$ and gradually decreasing $\mathrm{CMR}_{\mathrm{Glc}} \mathrm{J}_{\mathrm{Lac}}$ and $\mathrm{CBF}$ under a high-frequency stimulation (e.g., 8 Hz) (Prichard et al., 1991; Gjedde and Marrett, 2001; Mintun et al., 2002; Vlassenko et al., 2006; Lin et al., 2009). Consequently, $\% \Delta \mathrm{CBF}$ and $\% \Delta \mathrm{CMRO}_{2}$ were re-coupled (the coupling ratio, $n=\% \Delta \mathrm{CBF} / \% \Delta \mathrm{CMRO}_{2}$, decreased from 8 to 2 ) as stimulation continued. However, inconsistent observations have also been reported in prior fMRI studies (Hoge et al., 1999) that the coupling ratio already reached to $2-3$ during acute, transient visual stimulation (approximately 20-60 s). The discrepancy could be due to the stimulation frequency or to the modeling strategy used in the studies. For example, a low coupling ratio (2-3) was observed with low frequencies (e.g., $1-4 \mathrm{~Hz}$ ), while a high ratio (6-10) was observed with high frequencies, during short-term visual stimulation (20 s to $6 \mathrm{~min}$ ) (Lin et al., 2008). As a result, ratio of $2-3$ is 
expected if low frequencies are used. Details of the discrepancy regarding modeling strategy can be found in our previous publication (Lin et al., 2009).

Metabolic physiology and functional neuroimaging are highly complementary fields of inquiry. An in-depth understanding of neuronal metabolic physiology will enhance the interpretation of functional neuroimaging research, and vice versa. The evidence above addressed the cooperation of functional neuroimaging and metabolic physiology in understanding the coordination between neurovasculature, neurons and astrocytes during steady-state. For future studies, more effort should be invested in exploring their coordination during transient state. For instance, the dynamic mechanism of the BOLD signal may be explained by the asynchrony of task-induced neuronal and glial responses, as follows. Because neuronal responses precede those of astrocytes (approximately $4 \mathrm{~s}$; Schummers et al., 2008), the increase in oxidative metabolism precedes that of non-oxidative metabolism (Kasischke et al., 2004) and, consequently, the $\mathrm{CBF}$ response. $\mathrm{CMRO}_{2}$ increases that precede CBF increases result in the "initial dip" of the BOLD signal (Menon et al., 1995; Malonek and Grinvald, 1996). Astrocytic activity, following the neuronal activity, results in a significant increase in $\mathrm{CBF}$ and, consequently, the increase in BOLD signal. On the other hand, the post-stimulus "undershoot" phenomenon (the negative signal following the cessation of stimulation; Buxton et al., 1998; Mandeville et al., 1999; Lu et al., 2004) observed in BOLD and CBF signals contradicts the notion of "watering the entire garden for the sake of one thirsty flower" (Malonek and Grinvald, 1996), but may pave a way for further understanding the temporal relationship between the neuronal and astrocytic activities after the stimulus termination.

Further developments in neuroimaging techniques and metabolic theories are crucial for future research. As regards neuroimaging techniques, it would be important to determine quantitative ATP production, $\mathrm{CMRO}_{2}$ and $\mathrm{CMR}_{\mathrm{Glc}}$ using other $\mathrm{MR}$ methods, such as Phosphorous-31, Oxygen-17 and Carbon-13 MRS, respectively. In addition, direct observations of the differential roles of neurons and astrocytes associated with ATP-CMRO 2 and CBF-lactate couples would be significant. Concurrent MRI, MRS and optical imaging measurements may help achieve this goal. As regards metabolic physiology, theories of neurovascular and neurometabolic mechanisms are evolving. Some issues proposed by the ANLS model need further investigation. For example, whether the lactate transferred to neurons as a fuel substrate is from astrocytic or neuronal activity, and whether lactate is the preferential substrates of neurons for neurotransmission-related energy needs is still under debate. Further, whether the small increase in $\mathrm{CMRO}_{2}$ during neuronal activation is due to the small fraction of glucose undergoing oxidative

\section{REFERENCES}

Brand, M. D. (2005). The efficiency and plasticity of mitochondrial energy transduction. Biochem. Soc. Trans. 33, 897-904.

Buxton, R. B., Wong, E. C., and Frank, L. R. (1998). Dynamics of blood flow and oxygen metabolism during brain activation: the balloon model. Magn . Reson. Med. 39, 855-864.
Fox, P.T., and Raichle, M.E. (1986). Focal physiological uncoupling of cerebral blood flow and oxidative metabolism during somatosensory stimulation in U.S.A. 83, 1140-1144.

Fox,P.T., Raichle,M.E., Mintun, M.A., and Dence, C. (1988). Nonoxidative glucose consumption during focal physiologic neural activity. Science 241, 462-464. human subjects. Proc. Natl. Acad. Sci.

phosphorylation or due to other reasons is controversial. Brand proposed that ATP can be synthesized by means of deactivation of mitochondrial uncoupling protein (UCP) to elevate oxidative phosphorylation without raising oxygen consumption, which may provide an alternative explanation for relative low levels of $\mathrm{CMRO}_{2}$ changes during brain activation (Brand, 2005).

The concurrent developments of neuroimaging methods and physiological theories will also pave the way for the investigation of cerebral hemodynamics and metabolism in disease states. For example, the most well-documented example of a mitochondrialfailure-based neurodegenerative disorder is Huntington's disease (HD) (Jenkins et al., 1993; Koroshetz et al., 1997). Nonetheless, Powers et al. (2007) recently reported PET evidence that the welldocumented regional decreases in $\mathrm{CMR}_{\mathrm{Glc}}$ associated with $\mathrm{HD}$ are not accompanied by comparable decreases in $\mathrm{CMRO}_{2}$, which led them to conclude that HD is not a mitochondrial disorder. (We would argue, however, that this conclusion is not yet warranted, as Powers' study did not include a challenge, in which neuronal activation was used to elevate $\mathrm{MRO}_{2}$ above resting levels). Similar neuroimaging approaches may apply to other neurodegenerative disorders, including Alzheimer's disease (AD), Down's Syndromerelated $\mathrm{AD}$ and Parkinson's disease $(\mathrm{PD})$, to investigate possible underlying metabolic dysfunctions of those diseases. In addition to human studies, animal models also play an essential role since they allow more flexibility for neurovascular-metabolic coupling investigations of both normal and disease states. For example, treatments of rapamycin (a drug developed as an immunosuppressant) and calorie restriction are currently only available for animals in aging and AD studies (Van Remmen et al., 2001; Richardson, 2009; Spilman et al., 2010). Neuroimaging methods can help clarify the pathophysiology of these diseases and identify the mechanism of action of these effective treatments (in aging and AD) by defining the correlation between changes in $\mathrm{CBF}, \mathrm{CMRO}_{2}, \mathrm{CMR}_{\mathrm{Glc}}$ and ATP.

In summary, we addressed our perspective on the interplay of metabolic physiology and functional neuroimaging over the past century; the replacement of the Roy-Sherrington principle by the ANLS model; and, the future direction of the developments of neuroimaging techniques and physiological theories. These developments should provide the bases for predicting causes and consequences of deregulation in neurological diseases, including neurodegenerative disorders, in the near future.

\section{ACKNOWLEDGMENTS}

This work was supported by National Institute of Health (NIH) and UTHSCSA General Clinical Research Center (GCRC) grants M01 RR01346.

Frackowiak, R. S., Lenzi, G. L., Jones, T., and Heather, J. D. (1980). Quantitative measurement of regional cerebral blood flow and oxygen metabolism in man using $15 \mathrm{O}$ and positron emission tomography: theory, procedure, and normal values. J. Comput. Assist. Tomogr. 4, 727-736.

Frahm, J., Kruger, G., Merboldt, K D., and Kleinschmidt, A. (1996).
Dynamic uncoupling and recoupling of perfusion and oxidative metabolism during focal brain activation in man. Magn. Reson. Med. 35, 143-148.

Fulton, J. (1928). Observation upon the vascularity of the human occipital lobe during visual activity. Brain 51, 310-320.

Gjedde, A. (1997). “The relation between brain function and cerebral blood flow 
and metabolism," in Cerebrovascular Disease, eds H. Bajter and L. Caplan (Philadelphia, PA: Lippincott-Raven Publishers), 23-40.

Gjedde, A., and Marrett, S. (2001). Glycolysis in neurons, not astrocytes, delays oxidative metabolism of human visual cortex during sustained checkerboard stimulation in vivo. J. Cereb. Blood Flow Metab. 21, 1384-1392.

Hoge, R. D., Atkinson, J., Gill, B., Crelier, G. R., Marrett, S., and Pike, G. B. (1999). Investigation of BOLD signal dependence on cerebral blood flow and oxygen consumption: the deoxyhemoglobin dilution model. Magn. Reson. Med. 42, 849-863.

Hyder, F., Patel,A. B., Gjedde, A., Rothman, D. L., Behar, K. L., and Shulman, R. G. (2006). Neuronal-glial glucose oxidation and glutamatergic-GABAergic function. J. Cereb. Blood Flow Metab. 26, 865-877.

Jenkins, B. G., Koroshetz, W. J., Beal, M. F., and Rosen, B. R. (1993). Evidence for impairment of energy metabolism in vivo in Huntington's disease using localized 1H NMR spectroscopy. Neurology 43, 2689-2695.

Kasischke, K.A., Vishwasrao, H. D., Fisher, P. J., Zipfel, W. R., and Webb, W. W. (2004). Neural activity triggers neuronal oxidative metabolism followed by astrocytic glycolysis. Science 305 , 99-103.

Kety, S. S., and Schmidt, C. F. (1948). The effects of altered arterial tensions of carbon dioxide and oxygen on cerebral blood flow and cerebral oxygen consumption of normal young men. J. Clin. Invest. 27, 484-492.

Koroshetz, W. J., Jenkins, B. G., Rosen, B. R., and Beal, M. F. (1997). Energy metabolism defects in Huntington's disease and effects of coenzyme Q10. Ann. Neurol. 41, 160-165.

Kwong, K. K., Belliveau, J. W., Chesler, D. A., Goldberg, I. E., Weisskoff, R. M., Poncelet, B. P., Kennedy, D. N., Hoppel, B. E., Cohen, M. S., Turner, R., Cheng, H. M., Brady, T. J., and Rosen, B. R. (1992). Dynamic magnetic resonance imaging of human brain activity during primary sensory stimulation. Proc. Natl. Acad. Sci. U.S.A. 89, 5675-5679.

Landau, W. M., Freygang, W. H. J., Roland, L. P., Sokoloff, L., and Kety, S. S. (1955). The local circulation of the living brain: values in the unanesthetized and anesthetized cat. Trans. Am. Neurol. Assoc. 80, 125-129.

Lin, A.-L., Fox, P. T., Hardies, J., Duong, T. Q., and Gao, J.-H. (2010). Nonlinear coupling between cerebral blood flow, oxygen consumption, and ATP production in human visual cortex. Proc. Natl. Acad. Sci. U.S.A. 107, 8446-8451.

Lin, A.-L., Fox, P. T., Yang, Y., Lu, H., Tan, L.-H., and Gao, J.-H. (2009). Timedependent correlation of cerebral blood flow with oxygen metabolism in activated human visual cortex as measured by fMRI. Neuroimage 44, 16-22.

Lin, A.-L., Fox, P. T., Yang, Y., Lu, H., Tan, L.-H., and Gao, J.-H. (2008). Evaluation of MRI models in the measurement of $\mathrm{CMRO} 2$ and its relationship with CBF. Magn. Reson. Med. 60, 380-389.

Lu, H., Golay, X., Pekar, J. J., and Van Zijl, P. C. (2004). Sustained poststimulus elevation in cerebral oxygen utilization after vascular recovery. J. Cereb. Blood Flow Metab. 24, 764-770.

Malonek, D., and Grinvald, A. (1996). Interactions between electrical activity and cortical microcirculation revealed by imaging spectroscopy: implications for functional brain mapping. Science 272, 551-554.

Mandeville, J. B., Marota, J. J., Ayata, C., Zaharchuk, G., Moskowitz, M. A., Rosen, B. R., and Weisskoff, R. M. (1999). Evidence of a cerebrovascular postarteriole windkessel with delayed compliance. J. Cereb. Blood Flow Metab. 19, 679-689.

Menon, R. S., Ogawa, S., Hu, X., Strupp, J. P., Anderson, P., and Ugurbil, K. (1995). BOLD based functional MRI at 4 Tesla includes a capillary bed contribution: echo-planar imaging correlates with previous optical imaging using intrinsic signals. Magn. Reson. Med. 33, 453-459.

Mintun, M. A., Raichle, M. E., Martin, W. R., and Herscovitch, P. (1984). Brain oxygen utilization measured with $\mathrm{O}-15$ radiotracers and positron emission tomography. J. Nucl. Med. 25, 177-187.

Mintun, M. A., Vlassenko, A. G., Rundle, M. M., and Raichle, M. E. (2004). Increased lactate/pyruvate ratio augments blood flow in physiologically activated human brain. Proc. Natl. Acad. Sci. U.S.A. 101, 659-664.

Mintun, M.A., Vlassenko,A. G., Shulman, G. L., and Snyder, A. Z. (2002). Timerelated increase of oxygen utilization in continuously activated human visual cortex. Neuroimage 16, 531-537.

Mosso, A. (1881). Ueber den Kreislauf des Blutes im menschlichen Gehirn. Leipzig: Verlag von Veit.
Ogawa, S., Tank, D. W., Menon, R., Ellermann, J. M., Kim, S. G., Merkle, H., and Ugurbil, K. (1992). Intrinsic signal changes accompanying sensory stimulation: functional brain mapping with magnetic resonance imaging. Proc. Natl. Acad. Sci. U.S.A. 89, 5951-5955.

Pellerin, L., and Magistretti, P. J. (1994). Glutamate uptake into astrocytes stimulates aerobic glycolysis: a mechanism coupling neuronal activity to glucose utilization. Proc. Natl. Acad. Sci. U.S.A. 91, 10625-10629.

Phelps, M. E., and Mazziotta, J. C. (1985). Positron emission tomography: human brain function and biochemistry. Science 228, 799-809.

Posner, M. I., and Raichle, M. E. (1994) Images of Mind. New York: Scientific American Library.

Powers, W. J., Videen, T. O., Markham, J. McGee-Minnich, L., Antenor-Dorsey, J. V., Hershey, T., and Perlmutter, J. S. (2007). Selective defect of in vivo glycolysis in early huntington's disease striatum. Proc. Natl. Acad. Sci. U.S.A 104, 2945-2949.

Prichard, J., Rothman, D., Novotny, E., Petroff, O., Kuwabara, T., Avison, M., Howseman, A., Hanstock, C. and Shulman, R. (1991). Lactate rise detected by $1 \mathrm{H}$ NMR in human visual cortex during physiologic stimulation. Proc. Natl. Acad. Sci. U.S.A. 88, 5829-5831.

Raichle, M. E., Martin, W. R., Herscovitch, P., Mintun, M. A., and Markham, J. (1983). Brain blood flow measured with intravenous H2(15)O. II. Implementation and validation. $J$. Nucl. Med. 24, 790-798.

Reivich, M., Kuhl, D., Wolf, A., Greenberg, J., Phelps, M., Ido, T., Casella, V., Fowler, J., Hoffman, E., Alavi, A. Som, P., and Sokoloff, L. (1979). The [18F]fluorodeoxyglucose method for the measurement of local cerebral glucose utilization in man. Circ. Res. 44, 127-137.

Richardson, R. B. (2009). Ionizing radiation and aging: rejuvenating an old idea. Aging 1, 887-902.

Roy, C. S., and Sherrington, C. S. (1890) On the regulation of the blood-supply of the brain. J. Physiol. (Lond.) 11, 85-108.

Schmidt,C.F., and Kety, S.S. (1947). Recent studies of cerebral blood flow and cerebral metabolism in man. Trans. Assoc. Am. Physicians 60, 52-58.

Schummers, J., Yu, H., and Sur, M. (2008) Tuned responses of astrocytes and their influence on hemodynamic signals in the visual cortex. Science 320 , 1638-1643.

Siesjo, B. (1978). Brain Energy Metabolism. New York: Wiley, 101-110.

Sokoloff, L., Reivich, M., Kennedy, C., Des Rosiers, M. H., Patlak, C. S., Pettigrew, K. D., Sakurada, O., and Shinohara, M. (1977). The [14C] deoxyglucose method for the measurement of local cerebral glucose utilization: theory, procedure, and normal values in the conscious and anesthetized albino rat. J. Neurochem. 28, 897-916.

Spilman, P., Podlutskaya, N., Hart, M. J., Debnath, J., Gorostiza, O., Bredesen, D., Richardson, A., Strong, R., and Galvan, V.(2010). Inhibition of mTOR by rapamycin abolishes cognitive deficits and reduces amyloid-beta levels in a mouse model of Alzheimer's disease. PLoS ONE5, e9979. doi: 10.1371/journal.pone.0009979.

Vafaee, M. S., and Gjedde, A. (2000). Model of blood-brain transfer of oxygen explains nonlinear flow-metabolism coupling during stimulation of visual cortex. J. Cereb. Blood Flow Metab. 20, 747-754.

Van Remmen,H.,Guo,Z., and Richardson, A. (2001). The anti-ageing action of dietary restriction. Novartis Found. Symp. 235, 221-230.

Vlassenko, A. G., Rundle, M. M., Raichle, M. E., and Mintun, M. A. (2006). Regulation of blood flow in activated human brain by cytosolic NADH/ NAD + ratio. Proc. Natl. Acad. Sci. U.S.A. 103, 1964-1969.

Conflict of Interest Statement: The authors declare that the research was conducted in the absence of any commercial or financial relationships that could be construed as a potential conflict of interest.

Received: 10 February 2010; paperpending published: 15 April 2010; accepted: 01 July 2010; published online: 21 July 2010.

Citation: Lin A-L, Gao J-H, Duong TQ and Fox PT (2010) Functional neuroimaging: $a$ physiological perspective. Front. Neuroenerg. 2:17. doi: 10.3389/fnene.2010.00017 Copyright (c) 2010 Lin, Gao, Duong and Fox. This is an open-access article subject to an exclusive license agreement between the authors and the Frontiers Research Foundation, which permits unrestricted use, distribution, and reproduction in any medium, provided the original authors and source are credited. 